Int. J. Dev. Biol. 48: 629-635 (2004)

doi: $10.1387 / \mathrm{ijdb} .041898 \mathrm{sf}$

Original Article

\title{
$X$-chromosome inactivation in differentiating mouse embryonic stem cells carrying X-linked GFP and lacZ transgenes
}

\author{
SHIRIN FARIVAR ${ }^{1}$, SHINPEI YAMAGUCHI ${ }^{1}$, MICHIHIKO SUGIMOTO ${ }^{1,3}$ and NOBUO TAKAGI*,1,2 \\ ${ }^{1}$ Graduate School of Environmental Earth Science, Hokkaido University, Sapporo, Japan, ${ }^{2}$ Hokusei Gakuen University, Sapporo, Japan and \\ ${ }^{3}$ BioResource Center, Riken Tsukuba Institute, Ibaraki, Japan
}

\begin{abstract}
Three new female ES cell lines (GLM1, GLP1 and GLP2) were established from mouse embryos carrying GFP (green fluorescent protein) and HMG-lacZ transgenes on one of two $X$ chromosomes in cis. Using these cell lines, we studied the temporal relationships among three events relevant to $X$-chromosome inactivation: replication asynchrony of the $X$ chromosome, and quenching of GFP fluorescence and $\beta$-galactosidase ( $\beta$-gal) activity, during cell differentiation induced by embryoid body (EB) formation and retinoic acid (RA) treatment. In embryoid bodies adhering to the bottom of culture dishes, GFP-negative cells appeared first in the peripheral outgrowths 4 days after the initiation of EB formation, followed about 24 hours later by the appearance of cells negative for $\beta$-gal and those having a single allocyclic $X$ chromosome. Although the frequency of cells with an allocyclic $X$ chromosome could reach $80 \%$ in adherent embryoid bodies, it tended to remain low and variable in embryoid bodies maintained in suspension. In spite of apparently parallel extinction of GFP and lacZ in embryoid bodies, their concurrent occurrence did not always characterize RA-induced differentiation. Moreover, an allocyclic X chromosome was identified in not more than 20 percent of informative metaphase cells up to 10 days after initiation of RA treatment. These findings suggest that RA-induced differentiation of female ES cells does not always accompany $\mathrm{X}$-inactivation.
\end{abstract}

KEY WORDS: ES cell, embryoid body, lacZ, GFP, X-inactivation

\section{Introduction}

In early embryogenesis of female mammals, one of the two $\mathrm{X}$ chromosomes is randomly inactivated, which results in compensation of the difference in the $\mathrm{X}$-linked gene dosage between males and females (Lyon, 1961). While a number of recent studies have focused on Xist and other genes which have relevance to the initiation of X-inactivation (reviewed by Avner and Heard, 2000, Plath etal., 2002), little is known about the manner of $X$-inactivation during the early stages of embryogenesis. It is extremely difficult to analyze initiation of $X$-inactivation in vivo, since it occurs at different times in different tissues of small embryos grown in utero. The situation is complicated in the mouse because inactivation is imprinted in the trophectoderm and primitive endoderm selectively silencing the paternally inherited $X\left(X^{P}\right)$ chromosome and it is random in the epiblast, which gives rise to the entire fetus (reviewed by Heard et al., 1999).

Monk and Harper (1978) and Monk (1981) first noticed the close temporal correlation between differentiation of tissues such as the trophectoderm and primitive endoderm and the reduction by half of the relative activity of $\mathrm{X}$-linked HPRT. The tight connection be- tween X-inactivation and cell differentiation was supported by Martin et al. (1978), who showed that the induction of cell differentiation in vitroleads to $\mathrm{X}$-inactivation in an XX embryonal carcinoma cell line, LT-1. Cell differentiation may be a necessary (Wutz and Jaenisch 2002) but insufficient correlate of $X$-inactivation, because apparently normal morphological differentiation of LT-1 aggregates without concomitant $X$-inactivation can occur (Takagi and Martin, 1984). ES cells differentiating in vitro have been widely used as a model system for analyzing the molecular mechanisms of random X-inactivation. However, not much is known about the details of this model system. The parietal or visceral endoderm appears first in the in vitrodifferentiation system involving embryoid body formation. The paternally inherited $X$ chromosome is preferentially inactivated in these cell types in vivo(West et al., 1978). In agreement with this observation, a cytogenetic study of differentiating ES cells having a Robertsonian X-autosome translocation showed that the paternally inherited $X$ chromosome is preferentially

Abbreviations used in this paper: EB, embryoid body; ES, embryonic stem; GFP, green fluorescent protein; RA, retinoic acid.

*Address correspondence to: Dr. NobuoTakagi. Hokusei Gakuen University, 3-1 Oyachi Nishi 2, Atsubetsu-ku, Sapporo 004-8631, Japan. e-mail: ntakagi@u01.gate01.com 
inactivated (Tada et al., 1993). This finding, though not reproduced in a subsequent study in the endoderm layer separated from individual cystic embryoid bodies (Sado etal., 1996), deserves reappraisal making use of newer methods. Another representative method of ES cell differentiation is retinoic acid treatment (Strickland and Mahdavi, 1978). The occurrence of X-inactivation has been reported in P10 embryonal carcinoma cell line (Paterno and McBurney, 1985) and some ES cell lines (Heard etal., 2001; Wutz and Jaenisch, 2002), but the efficiency has not been critically tested.

Exogenous reporter genes such as the bacterial lacZ and jellyfish GFP genes, inserted into the $\mathrm{X}$ chromosome have been used to visualize $X$-inactivation in individual cells and have provided various new findings (Tan et al., 1993; Tam et al., 1994; Jamieson et al., 1998; Sugimoto et al., 2000; Okamoto et al., 2000; Tada etal., 2000; Sado etal., 2001; Takagi etal., 2002). The GFPtransgene has been used to monitor changes in X-chromosome activity in situwithout killing the cell. The X-linked HMG-lacZ transgene, on the other hand, has made the detailed histological analysis of X-inactivation at the cell or tissue level possible after fixation and staining. In this study we aimed to establish new ES cell lines from female mouse embryos carrying both X-linked GFPand HMG-lacZtransgenes to evaluate their usefulness for the analysis of $X$-inactivation. Our study using these newly established ES cell lines, showed that the $\mathrm{X}$-inactivation in differentiating embryoid bodies is mostly random but the possibility that there is occasional cell type-specific non-random choice is not completely ruled out. Furthermore, our findings suggested that the cell differentiation induced by retinoic acid does not always accompany typical X-inactivation.

\section{Results}

Three XX ES cell lines were established in this study. One (GLM1) carried both transgenes (GFPand HMG-lacZ) on the maternally inherited X-chromosome and the remaining two (GLP1 and GLP2) carried both transgenes on the paternally derived X-chromosome. These ES cell lines were fully fluorescent irrespective of the parental origin of the GFPtransgene. Expression of the other X-linked transgene, HMG-lacZ, was unusually high in these cell lines, as indicated by the fact that undifferentiated cells were heavily stained within less than half an hour. The karyotypes of these cell lines were apparently normal, with 40 chromosomes including two synchronously replicating X-chromosomes. The frequency of tetraploid cells was fairly low.

\section{Embryoid body formation}

Differentiation experiments were carried out mainly with the GLM1 cell line. Embryoid bodies were formed as described in Materials and Methods. Numerous cell aggregates were formed after overnight incubation of the cell suspension in bacteriological Petri dishes. A high proportion of the aggregates became surrounded with endodermal cells within 3 days. The aggregates
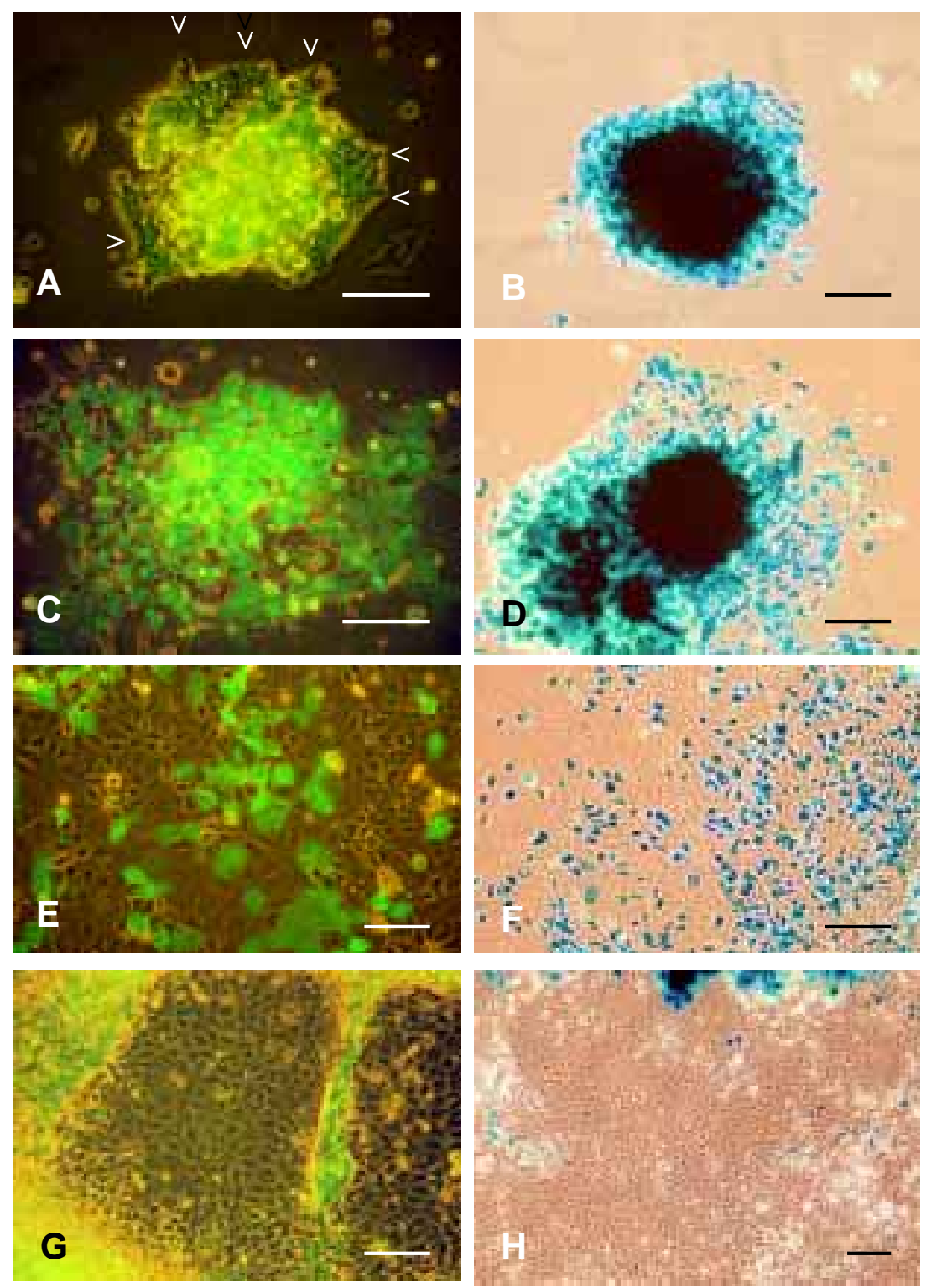

Fig. 1. $X$ chromosome inactivation revealed by extinction of GFP $(A, C, E, F)$ and lacZ $(B, D, F, H)$ in embryoid bodies attached to tissue culture dishes. Some peripheral cells (arrowheds) are already GFP-negative one day after attachment (day 4 of differentiation) suggesting the occurrence of $X$-inactivation (A), although all cells are still positive for $\beta$-gal (B). GFP-negative or $\beta$-gal-negative cells are found in every embryoid body at day 5 (C,D). The expression pattern of the transgene is mostly consistent with random X-inactivation at day 6 of differentiation (E,F), but the large patches of cells uniformly negative for GFP fluorescence $(\mathbf{G})$ and $\beta$-gal $(\mathbf{H})$ are not rare, suggesting the occurrence of non-random $X$ inactivation. Bar, $100 \mu \mathrm{m}$.

transferred to tissue culture dishes 3 days after the initiation of differentiation adhered to the bottom of the dishes and the outer cells began to migrate away from and form a confluent halo around the embryoid body core within 24 hours. Embryoid bodies kept in suspension grew larger and some of them became cystic by eccentric cavitation. By day 9 in suspension culture, some of cystic embryoid bodies had expanded greatly, resulting in balloon-like cysts as reported for EC and ES cell lines (Martin et al., 1977; Sado et al., 1996). 


\section{Evaluation of X-chromosome activity by GFP fluorescence}

The GFP transgene driven by the chicken $\beta$-actin promoter is ubiquitously expressed from the early embryonic stage to adulthood in mice. All GLM1 cells continued to be fluorescent for the first 2 days after the initiation of embryoid body formation. By day 3 , some cells in the outer endoderm layer became non-fluorescent. Most embryoid bodies had at least some non-fluorescent cells in the outer layer by day 4 (Fig. 1A), but the frequency of GFP-negative cells varied considerably among different embryoid bodies suggesting the asynchrony in the initiation of $\mathrm{X}$-inactivation among different embryoid bodies and different cells in a single embryoid body. Although quantification was difficult in the regions crowded with differentiating cells, the heterogeneity increased by day 5 and 6 . In certain areas, the fluorescence patterns were consistent with random inactivation (Fig. $1 \mathrm{C}, \mathrm{E}$ ), but in other areas they were apparently at variance with random inactivation (Fig. 1G). The uniform patches were too large to have been derived from a single progenitor cell after X-inactivation. The cells shown in Fig. $1 \mathrm{G}$ morphologically resemble the endoderm of early embryonic stages. If they are the visceral endoderm, their behavior is unusual because the maternal $X$ chromosome is inactivated in this cell lineage, in which the paternal $X$ is usually inactivated preferentially in vivo. Another unusual feature is that these endoderm cells were derived from ES cells of the epiblast lineage, where random inactivation usually takes place. Although non-fluorescent cells increased in frequency until day 8 , cells at the center of each embryoid body still remained brightly fluorescent.

\section{lacZ expression in adherent embryoid bodies}

As in the case of GFP fluorescence, the appearance of cells negative for $\beta$-gal suggests the initiation of $X$-inactivation. $\beta$-gal activity was extremely high in undifferentiated GLM1 cells, as mentioned earlier and the cells were stained heavily with X-Gal in a short period of time. Not only the cell but also the staining buffer became deep bluegreen within 30 minutes. In embryoid bodies adhering to the dish, $\beta$-gal-negative cells were not found at day 4 (Fig. $1 \mathrm{~B})$, but they began to appear at the peripheral region by day 5 (Fig. 1D), 2 days after the transfer to tissue culture dishes. The frequency of $\beta$-gal-negative cells increased in frequency thereafter, like that of GFP-negative cells, with substantial asynchrony among different embryoid bodies. Although patches of nearly equal proportion of $\beta$-galpositive and $\beta$-gal-negative cells were frequently found, consistent with random $X$-inactivation (Fig. 1F), large patches of uniformly $\beta$-gal-negative were not rare (Fig. $1 \mathrm{H})$. $\beta$-gal-negative cells were, in general, GFP-negative and the extinction of GFP fluorescence slightly preceded the loss of $\beta$-gal activity.

\section{Asynchronously replicating X-chromosome in the em- bryoid body}

Replication asynchrony (allocycly) has been used as a potent indicator of $\mathrm{X}$-chromosome inactivation. The cytogenetically identified allocyclic X chromosomes were mostly late replicating, but a minority of them replicated earlier than other chromosomes in the cell. In the first experiment using the GLM1 cell line (Table 1), an allocyclic Xchromosome was found in $17 \%$ of metaphase cells prepared from day 4 embryoid bodies kept in suspension. The frequency increased gradually, reaching $39 \%$ at day 8 . An allocyclic X-chromosome was detected only in $10 \%$ of metaphase cells in adherent embryoid bodies at day 4, one day after transfer to the tissue culture dish. The frequency was lower in embryoid bodies in the
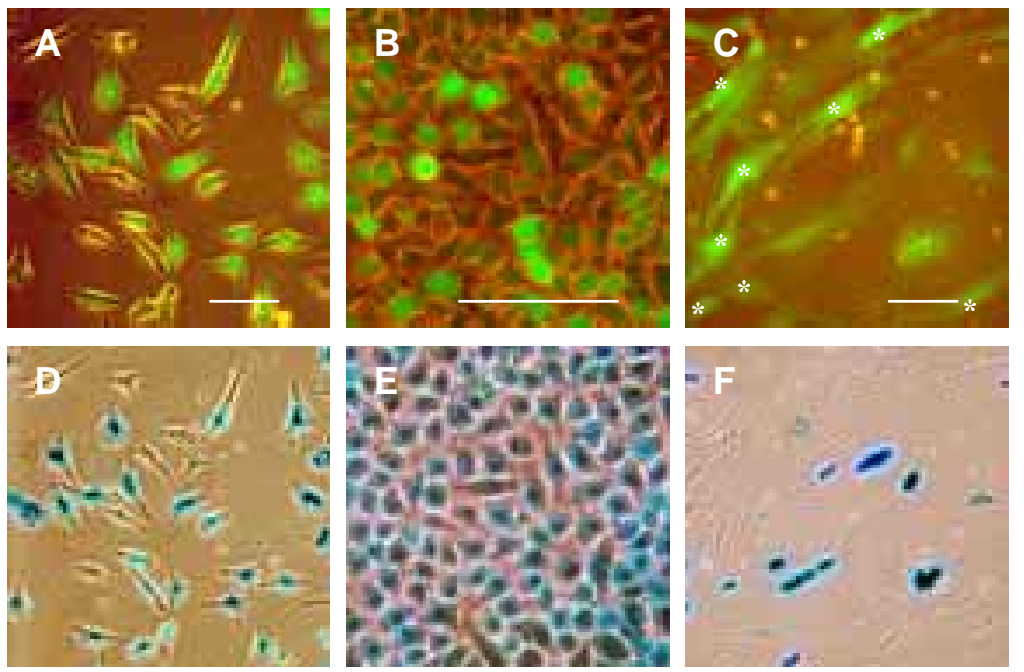

G

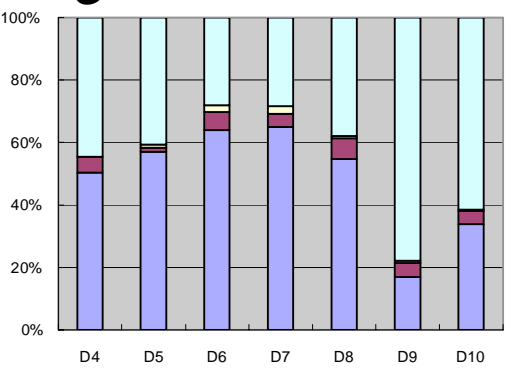

I

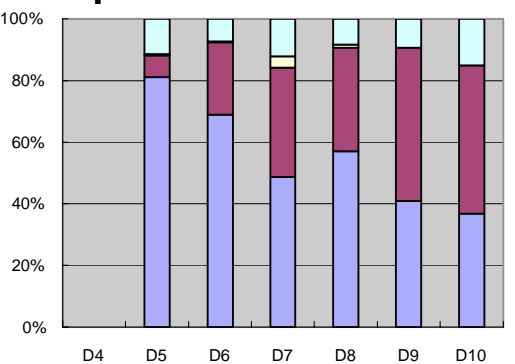

GFP+//gal +
H

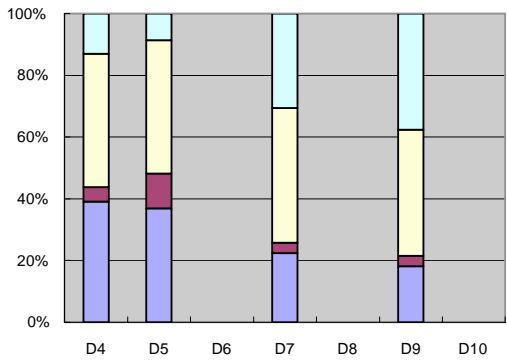

J

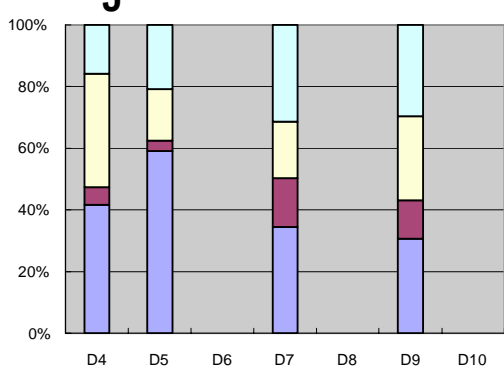

GFP-/Bgal+
GFP-/ßgal-
Fig. 2. Inconsistent loss of GFP and $\beta$-gal activity in differentiating GLM1 cells after exposure to retinoic acid. GFP-negative cells in (A) are consistently $\beta$-galnegative in (D) and vice versa. Not less than 40 endoderm cells in (B) are GFPnegative, but they are consistently $\beta$-gal-positive (E). In contrast, GFP-positive fibroblasts marked by asterisks in (C) are $\beta$-gal negative (F). Results of observations similar to those shown in (A-F) are summarized in (G-J). The proportion of four cell types with respect to the expression or non-expression of two transgenes varies greatly in four independent experiments. The initial cell number was $5 \times 10^{4}$ per $35 \mathrm{~mm}$ dish in $(G, 1)$, whereas it was $1.5 \times 10^{5}$ per $35 \mathrm{~mm}$ dish in $(\mathrm{H}, \mathrm{J})$ Cells were treated with retinoic acid in the absence of dibutyryl cAMP and isobutylmethylxanthine in (J). Bar, $100 \mathrm{~mm}$. 
adherent condition than in those in suspension at days 5 and 6 , but the situation was reversed by day 7 . Nearly $50 \%$ of metaphase cells had an allocyclic X-chromosome in adherent embryoid bodies at day 8 showing that the adherent condition accelerated cell differentiation and hence $\mathrm{X}$-inactivation.

In the second experiment, samples were taken from day 2 (Table 1) to determine the earliest time to turn the $\mathrm{X}$ chromosome allocyclic. In contrast to the previous experiment, the allocyclic $X$ chromosome was found neither in embryoid bodies in the adherent condition nor in those maintained in suspension until day 4 in this experiment, except in one cell found at day 2 (Table 1). An allocyclic $X$ chromosome was regularly found in adhering embryoid bodies from day 5 onwards and the frequency increased rapidly, attaining nearly $80 \%$ at day 8 . In embryoid bodies kept in suspension, however, no allocyclic X chromosome appeared until day 5 . Less than $10 \%$ of the metaphase cell had an allocyclic $X$ chromosome from day 6 to 8 .

The GLP1 and GLP2 cell lines showed essentially the same behavior as the GLM1 cell line (Table 1). In the case of GLP1, embryoid bodies were transferred to the tissue culture dishes 2 days after the initiation of differentiation.

\section{Asynchronous loss of GFP fluorescence and $\beta$-gal activity in GLM1 cells induced to differentiate by retinoic acid}

RA-induced cell differentiation proceeded quickly when the initial cell density was low and almost all cells were morphologically distinct from undifferentiated ES cells by day 3. GFP fluorescence and $\beta$-gal activity in individual cells were studied by comparing photographs of cells viewed with a fluorescence microscope and those of the same cells taken after X-Gal staining (Fig. 2 A-F). Before differentiation, all cells were positive for both GFP and $\beta$-gal $(\mathrm{GFP}+/ \beta-\mathrm{gal}+)$. Random $\mathrm{X}$-inactivation predicts that half the cells would become negative for both GFP fluorescence and X-Gal staining (GFP-/ $\beta$-gal-) at the expense of GFP+/ $\beta$-gal+ cells. GFP+/ $\beta$-gal- and GFP-/ $\beta$-gal+ cells would also appear transiently if the difference in the half-lives of GFP and $\beta$-gal is substantially large.

\section{TABLE 1}

\section{THE FREQUENCY OF METAPHASE CELLS HAVING AN ALLOCYCLIC X CHROMOSOME AFTER INITIATION OF DIFFERENTIATION IN THREE ES CELL LINES}

\begin{tabular}{|c|c|c|c|c|c|c|c|c|}
\hline & \multicolumn{8}{|c|}{$\%$ of metaphase cells with an allocyclic } \\
\hline & \multirow[b]{2}{*}{2} & \multicolumn{7}{|c|}{ X chromosome* at day: } \\
\hline & & 3 & 4 & 5 & 6 & 7 & 8 & 9 \\
\hline \multicolumn{9}{|l|}{ GLM1-Exp. 1} \\
\hline Suspended & - & - & 17 & 22 & 28 & 22 & 39 & 33 \\
\hline Adherent** & - & - & 10 & 13 & 14 & 27 & 51 & 48 \\
\hline \multicolumn{9}{|l|}{ GLM1-Exp. 2} \\
\hline Suspended & 1 & 0 & 0 & 0 & 7 & 6 & 8 & - \\
\hline Adherent ${ }^{\star *}$ & - & - & 0 & 12 & 28 & 48 & 79 & - \\
\hline \multicolumn{9}{|l|}{ GLP1 } \\
\hline Suspended & 1 & 1 & 1 & - & - & - & 8 & - \\
\hline Adherent ${ }^{\star \star \star}$ & - & 3 & 9 & - & - & - & 60 & - \\
\hline \multicolumn{9}{|l|}{ GLP2 } \\
\hline Suspended & - & - & - & 22 & 31 & 35 & 35 & 36 \\
\hline Adherent ${ }^{\star *}$ & - & - & 0 & 18 & 54 & 57 & 80 & 70 \\
\hline
\end{tabular}

\footnotetext{
* One hundred informative metaphase cells were evaluated at each time point.

** Embryoid bodies formed in suspension were transferred to tissue culture dishes at day 3 to further promote their differentiation.

${ }^{\star \star \star}$ Embryoid bodies were transferred to tissue culture dishes at day 2.
}

TABLE 2

\section{THE FREQUENCY OF METAPHASE CELLS HAVING AN ALLOCYCLIC X CHROMOSOME IN GLM1 CELLS TREATED WITH RETINOIC ACID}

\begin{tabular}{|c|c|c|c|c|c|c|c|}
\hline \multirow[t]{2}{*}{$\begin{array}{l}\text { Initial no. cells } \\
\text { per } 35 \mathrm{~mm} \text { dish }\end{array}$} & \multicolumn{7}{|c|}{$\begin{array}{c}\% \text { of metaphase cells with an asynchronously } \\
\text { replicating } X \text { chromosome at day: }\end{array}$} \\
\hline & 4 & 5 & 6 & 7 & 8 & 9 & 10 \\
\hline $1.5 \times 10^{5}$ & 1 & & 5 & & & 9 & \\
\hline $1.0 \times 10^{5}$ & 6 & - & 9 & - & - & 8 & - \\
\hline $5.0 \times 10^{4}$ & 4 & - & $5.6^{\star *}$ & - & - & - & - \\
\hline $1.0 \times 10^{5}$ & 1.0 & - & - & 17 & 9 & 18 & 19 \\
\hline
\end{tabular}

*100 metaphase cells were evaluated at each time point.

** Only 82 metaphase cells were available for observation.

As shown in Fig. $2 \mathrm{G}$, a mixture of GFP+/ $\beta$-gal+ and GFP-/ $\beta$-galcells (Fig. 2 A,D) predominated from day 5 to 10 , in agreement with random inactivation. Unexpectedly, however, results obtained in a replicate experiment (Fig. 2I) differed substantially from the above observation. Here, GFP-/ $\beta$-gal- cells were mostly replaced by GFP $+/ \beta$-gal- cells. The presence of these unusual cells suggests that RA-induced cell differentiation did not always accompany typical X-inactivation. Preliminary studies showed that the initial number of $5 \times 10^{4}$ cells per $35 \mathrm{~mm}$ dish was not enough for cytogenetic studies. Hence, we used $60 \mathrm{~mm}$ dishes with the initial cell number of $1.5 \times 10^{5}, 3 \times 10^{5}$ and $6 \times 10^{5}$ (corresponding to $5-15 \times$ $10^{4}$ cells per $35 \mathrm{~mm}$ dish) for the study of $X$ chromosome replication timing. As shown in Table 2 , an allocyclic $X$ chromosome was consistently found in a low proportion of metaphase cells at day 4. The frequency increased slightly thereafter, but it still remained less than $10 \%$ at day 9 (Table 2). No metaphase cell was found at day 9 in the case of the experiment with the lowest initial cell number of $1.5 \times 10^{5}$ per dish. A further experiment was carried out with the initial cell number of $1 \times 10^{6}$ per $60 \mathrm{~mm}$ culture dish. The allocyclic $\mathrm{X}$ chromosome was consistently detected at slightly higher frequencies, but not more than $20 \%$ (Table 2).

GFP and lacZ expression was also examined in parallel with replication asynchrony of the $X$ chromosome. In addition to GFP+/ $\beta$-gal + and GFP-/ $\beta$-gal- cells, substantial proportions of GFP-/ $\beta$ $\mathrm{gal}+$ or GFP+/ $\beta$-gal- cells were found in these experiments (Fig. $2 \mathrm{H})$. It appeared probable that the frequency of the four classes of cells differ in different cell types. GFP-/ $\beta$-gal+ cells, for example, were frequently found in endoderm (Fig. 2 B,E) and flat trophoblast-like cells, whereas GFP+/ $\beta$-gal- cells were not rare among a population of muscle cells or fibroblasts (Fig. 2 C,F). Slight differences in experimental conditions including the initial density of ES cells seeded, may have an effect on the proportion of various cell types and hence the proportion of the four classes of cells. No clear difference was found between RA-differentiated cells with or without (Fig. 2J) dibutyryl cAMP and isobutylmethylxanthine.

\section{Discussion}

The newly established ES cell line GLM1 has the GFPand lacZ transgenes on the maternally inherited $X$ chromosome. Individual cells are uniformly fluorescent and highly $\beta$-gal positive under undifferentiated conditions. We have never observed cells showing such high $\beta$-gal activity in mouse embryos carrying exactly the 
same transgene. This suggests the possibility that ES cells are different from any early embryonic cells in vivo.

Repeated experiments with the GLM1 ES cell line showed that the time needed for the first appearance of cells having an allocyclic $X$ chromosome and the subsequent increase in their frequency are not constant after the initiation of cell differentiation by the formation of cell aggregates and withdrawal of LIF, as reported for LT-1 embryonal carcinoma cell lines (Takagi and Martin, 1984). Slight changes in the initial conditions, though they have not been identified yet, seem to affect the subsequent initiation of $X$-inactivation. In spite of substantial variation, the present results showed that X-inactivation is induced fairly efficiently if embryoid bodies are allowed to adhere to the culture dish. The frequency of cells having an allocyclic $X$ chromosome consistently attained a peak 8 days after the initiation of differentiation, 5 days after transfer of embryoid bodies to the tissue culture dish. Mitotic index and the frequency of cells having an allocyclic $X$ chromosome declined thereafter if the cultures were not subcultured. This is one of most efficient systems available for in vitro induction of $X$-inactivation and the extinction of both the GFP and lacZ transgenes indicates the efficient occurrence of $X$-inactivation. The reason for the generally low and inconsistent $X$-inactivation in embryoid bodies maintained in suspension remains unknown. Most probably, differentiation is slow or arrested in a majority of embryoid bodies in view of the fact that usually only a minority of them grow in size and develop into the balloon-like cystic form. Histological sections of embryoid bodies revealed that tissue differentiation was inhibited in most embryoid bodies that differentiated a layer of distal endoderm on their surface by depositing a tough, thick sheet of Reichert membrane-like substance (data not shown). Extensive differentiation was found only in embryoid bodies that gave rise to the outer layer of visceral endoderm. Mitosis and cell differentiation must have been stimulated, on the other hand, in most embryoid bodies adhering to the tissue culture dish before the formation of such delimiting membranes.

One of our main interests was to delineate the temporal order of three events, the appearance of an allocyclic $X$ chromosome, quenching of GFP fluorescence and loss of $\beta$-gal activity in differentiating ES cells to assess their suitability as an indicator of $X$-inactivation. The allocyclic $X$ chromosome first appeared 5 days after the initiation of differentiation in the second experiment with GLM1 cells. By day 4, non-fluorescent cells had already appeared at the periphery of some embryoid bodies adhering to the dish, whereas no $\beta$-gal-negative cells were detected at this time. GFPnegative cells were observed by day 5 . Thus, quenching of GFP fluorescence slightly precedes loss of $\beta$-gal activity and the initiation of replication asynchrony, both of which occur almost simultaneously in differentiating embryoid bodies attached to the substratum. The slightly late extinction of $\beta$-gal activity may not be due to later inactivation but to a longer half-life of $\beta$-gal than GFP and/or excess $\beta$-gal content in undifferentiated ES cells. It remains unknown whether asynchronous replication in fact follows quenching of GFP fluorescence. There is a possibility that they occur almost simultaneously, but the cell having an allocyclic $X$ chromosome could not be detected by chance simply because only 100 informative metaphase cells were examined cytogenetically, whereas many more cells were scanned in the case of GFP fluorescence. Although we have to obtain more quantitative data on the increase of the frequency of GFP-negative and $\beta$-gal-negative cells in the course of ES cell differentiation, the extinction of both GFP and $\beta$ $\mathrm{gal}$ is a reliable indicator of $X$-inactivation in ES cells differentiating via embryoid body formation. It has been shown that $X$-inactivation involves - chromatin changes, including a series of histone modifications (Heard et al., 2002). Extinction of GFP transgenes may be used as a chronological marker of $\mathrm{X}$-inactivation.

Another point of interest is that there were large endodermal patches of uniformly $\beta$-gal-positive or $\beta$-gal-negative cells suggesting non-random inactivation, in addition to patches apparently consistent with random inactivation in adhering GLM1 embryoid bodies at day 6 onwards. Same patches were also uniformly GFPpositive or GFP-negative. An individual patch should contain about 64 cells by day 6 , if $X$ inactivation occurred at day 3 and the cell divided twice a day thereafter. In fact, 20 to 30 cells formed the patches of GFP-negative cells in the areas consistent with random inactivation at day 6 . It is not known whether the much larger patches detected are explained by chance inactivation of the same $X$ chromosome in some neighboring cells or by truly nonrandom inactivation. We cannot exclude the possibility that this was due to endoderm-specific behavior of the transgenes under study, because similar patches were found in an experiment using GLP1 and GLP2 ES cell lines carrying transgenes on the paternally derived X chromosome. Earlier, Tada et al. (1993) reported that Xchromosomes of newly established ES cell lines retain the "memory" of parental origin and that the paternal X-chromosome is preferentially inactivated in differentiating embryoid bodies. However, a later study (Sado et al., 1996) could not confirm this finding. Further scrutiny of the large patches showing uniform expression of GFP or $\beta$-gal may reveal the unique behavior of $X$ chromosomes in differentiating ES cells, especially those of the primitive endoderm cell lineage.

Most cells treated with RA were distinct from undifferentiated ES cells in morphology by day 3 . The persistence of GFP+/ $\beta$-gal- and GFP-/ $\beta$-gal+ cells even at 10 days after initiation of differentiation is at variance with chromosome-wide $X$-inactivation and suggests that RA-induced cell differentiation does not always accompany $X$ inactivation, although it remains unknown how the expression of only one of two transgenes on the same $X$ chromosome is suppressed. In agreement with this view, the chromosome replication banding study revealed that not more than $20 \%$ of metaphase cells had an allocyclic $X$ chromosome throughout the 10 day period of experiment. This cytogenetic finding may be interpreted in at least two different ways. In the first interpretation, $\mathrm{X}$-inactivation has not occurred in a large proportion of morphologically differentiated cells. In the second, a minority of mitotically active undifferentiated cells has outnumbered the mitotically less active differentiated cells at metaphase. Closer examination revealed that nests of cells with morphologically undifferentiated ES cells were present surrounded by differentiated cells even at 10 days after the initiation of RA-treatment, if the initial cell density was high, i.e., $3 \times 10^{5}$ or $6 \times 10^{5}$ per $60 \mathrm{~mm}$ dish. This persistent survival of undifferentiated ES cells in the presence of RA awaits full explanation in view of the rather efficient $X$-inactivation and implicitly cell differentiation, that occurred in adherent embryoid bodies. It is likely that the RA-induced mitotically quiescent differentiated cells play a role as an efficient feeder layer for cells that escaped differentiation and that those undifferentiated cells expanded even in the presence of RA. Those undifferentiated cells undoubtedly contributed to the high frequency of metaphase cells having no allocyclic $X$ chromo- 
some, but the occurrence of GFP+/ $\beta$-gal- and GFP-/ $\beta$-gal+ cells implies that they are not the only factor. It is tempting to speculate that these transgenes were repressed independently in certain proportion of cells that had not undergone $X$-inactivation. Alternatively, $\mathrm{X}$-inactivation was segmental rather than chromosomewide. In either case, further studies are necessary to evaluate whether RA-induced cell differentiation is a reliable model system for differentiation that occurs in early embryonic development.

\section{Materials and Methods}

\section{Mice}

Recombinant mice carrying GFP and HMG-lacZ transgenes in cis on the $X$ chromosome designated as GL mice (Takagi et al., 2002) were used to produce blastocysts for the establishment of new ES cell lines. These mice were derived by the intercrossing of $\mathrm{F} 1$ animals between the transgenic lines $\mathrm{H} 253$ and 152A. The H253 transgenic mouse line generated by Tan et al. (1993) carries DNA fragments containing the Escherichia coli lacZ gene. This X-linked transgene contains the promoter of a mouse house keeping gene, 3-hydroxy-3-methylglutaryl coenzyme A reductase (HMG $C O A$ ), linked to an SV40 T-antigen nucleus localization signal sequence. This $H M G$-lac $Z$ transgene encodes nucleus-localized $\beta$-galactosidase ( $\beta$ gal), which is readily detectable by X-Gal staining in individual nuclei. The transgene integrated at a region close to the proximal one-third of the $X$ chromosome (Tam et al., 1994) is subject to X-inactivation. The other transgenic line, 152A, which was derived from line 38 generated by Nakanishi et al. (2002), carries the Aeqeuorea victoria green fluorescent protein (GFP) coding sequence driven by the chicken $\beta$-actinpromoter and cytomegalovirus enhancer (Ikawa et al., 1995) inserted at the distal onethird of the X-chromosome. This ubiquitously expressed GFPtransgene is also subject to $X$-inactivation.

\section{Recovery and selection of embryos}

Embryos were flushed from the uteri of mice at the stage of fully grown blastocysts ( $3.5 \mathrm{dpc}$ ) with MEM. Fluorescent $(X X)$ and non-fluorescent $(X Y)$ blastocysts from the cross $129 / \mathrm{SvxGL}$ were separated under a fluorescence stereomicroscope using a mouth-controlled micropipet. Fluorescent embryos were then placed into collagen coated tissue culture dishes that contained a feeder layer of STO cells with DMEM plus $10 \%$ FBS and $1 \times 10^{3}$ $\mathrm{U} / \mathrm{ml}$ leukemia inhibitory factor (LIF). In the case of the cross GLx129/Sv, all recovered embryos were cultured.

\section{Establishment of ES cell lines}

ES cell lines were established according to standard procedures (Hogan et al., 1994). Briefly, some of the blastocysts attached to the bottom of culture dishes grew into an egg-cylinder-like structure within several days in culture. They were transferred to collagen-coated 24 well tissue culture plates containing an STO feeder layer after dispersal into aggregates of 56 cells with the aid of trypsin (0.25\%)-EDTA (0.04\%) and a narrow micropipet. ES-like colonies that emerged were reseeded into wells with a fresh feeder layer and finally transferred to $35 \mathrm{~mm}$ culture dishes to give permanent cell lines.

\section{Embryoid body formation}

ES cells grown to near confluence without an STO feeder layer were detached with trypsin-EDTA solution and about $10^{7}$ ES cells in DMEM without LIF were seeded into a $90 \mathrm{~mm}$ bacteriological Petri dish (to which these cells do not adhere). Cell aggregates that appeared overnight were transformed into simple embryoid bodies, consisting of an outer endoderm layer and the inner core, within a few days. Three days after the initiation of differentiation embryoid bodies were divided into two populations, one of which continued to be maintained in suspension in non-adherent bacteriological Petri dishes and the other of which was allowed to adhere to the bottom of tissue culture dishes to promote differentiation. The medium was changed every 2 days. Embryoid bodies that adhered to the substratum were used for the observation of GFP expression, $\beta$-gal activity and the $X$ chromosome replication pattern. The $\mathrm{X}$ chromosome replication pattern was studied in embryoid bodies maintained in suspension.

\section{Induction of differentiation by retinoic acid}

ES cells were seeded at a low density of $5 \times 10^{4}$ cells per 35 -mm collagencoated dish in DMEM with $20 \%$ FBS containing $5 \times 10^{-8} \mathrm{M}$ retinoic acid, $10^{-}$ ${ }^{4} \mathrm{M}$ dibutyryl cAMP and $10^{-4} \mathrm{M}$ isobutylmethylxanthine. The medium was replaced every 48 hours. For the study of $X$ chromosome replication, we used $60 \mathrm{~mm}$ dishes with initial cell numbers of $1.5 \times 10^{5}, 3 \times 10^{5}, 6 \times 10^{5}$ in order to obtain sufficient numbers of metaphase cells. The medium was changed daily. In one experiment, ES cells were treated with $5 \times 10^{-8} \mathrm{M}$ retinoic acid in the absence of dibutyryl cAMP and isobutylmethylxanthine.

\section{Cytogenetic analysis}

Cytogenetic analysis was carried out according to an established method of replication banding (Dutrillaux et al., 1973). Undifferentiated ES cells and growing embryoid bodies were allowed to incorporate 5-bromo2-deoxyuridine (BrdU: $100 \mu \mathrm{g} / \mathrm{ml}$ ) for 7 hours, including 1 hour in the presence of colcemide $(1 \mu \mathrm{g} / \mathrm{ml})$. The cells were harvested and treated with $0.5 \% \mathrm{KCl}$, fixed with $3: 1$ methanol/acetic acid fixative and air-dried on clean slide glasses. Slides stained with freshly prepared acridine orange solution were observed under a fluorescence microscope.

\section{Analysis of transgene expression}

GFP expression was examined with an Olympus inverted fluorescence microscope and photographs were taken with a Nikon digital camera. Cells growing attached to tissue culture dishes or embryoid bodies were fixed with $4 \%$ paraformaldehyde in PBS for 10 min on ice. Samples were washed in rinse buffer and then stained in a buffer containing 5-bromo-4-chloro-3indolyl- $\beta$-D-galactopyranoside (X-Gal), potassium ferrocyanide and potassium ferricyanide overnight at $37^{\circ} \mathrm{C}$ to assess $\beta$-gal activity.

\section{Acknowledgements}

We thank Dr. Yuji Goto for invaluable suggestions and encouragement during the course of this study. We wish to express our gratitude to Dr. Seong-Seng Tan, University of Melbourne and Dr. Masaru Okabe, Osaka University, for the transgenic mouse lines $\mathrm{H} 253$ and \#152A, respectively. Mice were bred at the Center for Experimental Plants and Animals of Hokkaido University. SF was a recipient of a Japanese Government (Monbusho) Fellowship. This study was supported by a Grant-in-Aid from the Japan Society for Promotion of Science.

\section{References}

AVNER, P. and HEARD, E. (2001). X-chromosome inactivation: counting, choice and initiation. Nature Reviews Genet. 2: 59-68.

CHAUMEIL, J., OKAMOTO, I., GUGGIARI, M. and HEARD, E. (2002). Integrated kinetics of $X$ chromosome inactivation in differentiating embryonic stem cells. Cytogenet. Genome Res. 99: 74-84.

DUTRILLAUX, B., LAURENT, C. and COUTURIER, J. (1973). Coloration des chromosomes humains par l'acridine orange après tritment par le 5 bromodeoxyuridine. CR Acad SC Paris 276: 3179-3181.

HEARD, E., CLERC, P. and AVNER, P. (1997). X-chromosome inactivation in mammals. Annu. Rev. Genet. 31: 571-610

HEARD, E., ROUGEULLE, C., ARNAUD, D., AVNER, P., ALLIS, C. D. and SPECTOR, D.L. (2001). Methylation of histone $\mathrm{H} 3$ at lys-9 is an early mark on the $X$ chromosome during $X$ inactivation. Cell 107: 727-738.

HOGAN, B., BEDDINGTON, R., COSTANTINI, F. and LACY E. (1994). Manipulating the mouse embryo. A laboratory manual. $2^{\text {nd }}$ ed. Cold Spring Harbor Laboratory, Plainsview, NY. P.497.

IKAWA, M., KOMINAMI, K., YOSHIMURA, Y., TANAKA, K., NISHIMUNE, Y. and OKABE, M. (1995). Green fluorescent protein as a marker in transgenic mice. Develop. Growth Differ. 37: 455-459. 
JAMIESON, R. V., TAN, S. S. and TAM, P. P. L. (1998). Retarded postimplantation development of $\mathrm{X} 0$ mouse embryos: impact of the parental origin of the monosomic X chromosome. Dev. Biol. 201: 13-25.

LYON, M. F. (1961). Gene action in the X-chromosome of the mouse (Mus musculus L.). Nature 190: 372-373.

MARTIN, G. R. and EVANS, M. J. (1975). Differentiation of clonal lines of teratocarcinoma cells: formation of embryoid bodies in vitro. Proc. Natl. Acad. Sci. USA72: 1441-1445.

MARTIN, G. R., WILEY, L. M. and DAMJANOV, I. (19779. The development of cystic embryod bodies in vitrofrom clonal teratocarcinoma stem cells. Dev. Biol.61:230244.

MONK, M. and HARPER, M. J. (1978). X-chromosome activity in preimplantation mouse embryos from XX and XO mothers. J. Embryol. Exp. Morph. 46: 53-64.

MONK M. (1981). A stem-line model for cellular and chromosomal differentiation in early mouse development. Differentiation 19: 71-76.

NAKANISHI, T., KUROIWA, A., YAMADA. S., ISOTANI, A., YAMASHITA, A., TAIRAKA, A., HAYASHI, T., TAKAGI. T., IKAWA, M., MATSUDA, Y. and OKABE, M. (2002). FISH analysis of 142 EGFP transgene integration sites into the mouse genome. Genomics 80: 564-574.

OKAMOTO, I., TAN, S. S. and TAKAGI, N. (2000). X-chromosome inactivation in XX androgenetic mouse embryos surviving implantation. Development 127: 41374145.

PATERNO, G. D. and MCBURNEY, M. W. (1985). X chromosome inactivation during induced differentiation of a female mouse embryonal carcinoma cell line. J. Cell Sci. 75: 149-163.

PLATH, K., MLYNARCZYK-EVANS, S., NUSINOW, D. A. and PANNING, B. (2002). $X i s t$ RNA and the mechanism of $\mathrm{X}$ chromosome inactivation. Annu. Rev. Genet. 36: $233-278$.

SADO, T., TADA, T. and TAKAGI, N. (1996). Mosaic methylation of Xistgene before chromosome inactivation in undifferentiated female mouse embryonic stem and embryonic germ cells. Dev. Dyn. 205: 421-434.

SADO, T., WANG, Z., SASAKI, H. and LI, E. (2001). Regulation of imprinted Xchromosome inactivation in mice by Tsix. Development 128: 1275-1286.

STRICKLAND, S. and MAHDAVI, V. (1978). The induction of differentiation in teratocarcinoma stem cells by retinoic acid. Cel/15: 393-403.
SUGIMOTO, M., TAN, S. S. and TAKAGI, N. (2000). X chromosome inactivation revealed by the $\mathrm{X}$-linked lac $Z$ transgene activity in periimplantation mouse embryos. Int. J. Dev. Biol. 44: 177-182.

TADA, T., OBATA, Y., TADA, M., GOTO, Y., NAKATSUJI, N., TAN, S. S. and TAKAGI, N. (2000). Imprint switching for non-random $X$-chromosome inactivation during mouse oocyte growth. Development 127: 3101-3105.

TADA, T., TADA, M. and TAKAGI, N. (1993). X chromosome retains the memory of its parental origin in murine embryonic stem cells. Development 119: 813-821.

TAKAGI, N. and MARTIN, G. R. (1984). Studies of the temporal relationship0 between the cytogenetic and biochemical manifestations of X-chromosome inactivation during the differentiation of LT-1 teratocarcinoma stem cells. Dev. Biol. 103: 425433.

TAKAGI, N. and SASAKI, M. (1975). Preferential inactivation of the paternally derived $X$-chromosome in the extraembryonic membranes of the mouse. Nature256: 640642.

TAKAGI, N., YAMAGUCHI, S., SUGIMOTO, M., ITO, M., TAN, S. S. and OKABE, M. (2002). Non-random $X$ chromosome inactivation in mouse embryos carrying Searle's $\mathrm{T}(\mathrm{X} ; 16) 16 \mathrm{H}$ translocation visualized using $\mathrm{X}$-linked lacZ and GFP tarnasgenes. Cytogenet. Genome Res. 99: 52-58.

TAM, P. P. L., WILLIAMS, E. A. and TAN, S. S. (1994). Expression of an X-linked HMG-lacZtransgene in mouse embryos; implication of chromosomal imprinting and lineage-specific X-chromosome activity. Dev. Genet. 15: 491-503.

TAN, S. S., WILLIAMS, E. A. and TAM. P. P. L. (1993). X chromosome inactivation occurs at different times in different tissues of the post-implantation mouse embryo. Nature Genet. 15: 170-174.

WEST, J. D., FRELS, W. I., CHAPMAN, V. M. and PAPAIOANNOU, V. E. (1977). Preferential expression of the maternally derived $\mathrm{X}$-chromosome in the mouse yolk sac. Cel/12: 873-882.

WUTZ, A. and JAENISCH, R. (2000). A shift from reversible to irreversible $X$ inactivation is triggered using ES cell differentiation. Mol. Ce//5: 695-705.

Received: May 2004 Reviewed by Referees: June 2004

Modified by Authors and Accepted for Publication: August 2004 Edited by: Goro Eguchi 